\title{
Bilayer Protograph Codes for Half-Duplex Relay Channels
}

\author{
Thuy Van Nguyen, Student Member, IEEE, Aria Nosratinia, Fellow, IEEE, and Dariush \\ Divsalar, Fellow, IEEE
}

\begin{abstract}
Despite encouraging advances in the design of relay codes, several important challenges remain. Many of the existing LDPC relay codes are tightly optimized for fixed channel conditions and not easily adapted without extensive re-optimization of the code. Some have high encoding complexity and some need long block lengths to approach capacity. This paper presents a high-performance protograph-based LDPC coding scheme for the half-duplex relay channel that addresses simultaneously several important issues: structured coding that permits easy design, low encoding complexity, embedded structure for convenient adaptation to various channel conditions, and performance close to capacity with a reasonable block length. The application of the coding structure to multi-relay networks is demonstrated. Finally, a simple new methodology for evaluating the end-to-end error performance of relay coding systems is developed and used to highlight the performance of the proposed codes.
\end{abstract}

\section{Index Terms}

Relay channel, LDPC codes, protograph codes, multiple-relay channel

\section{INTRODUCTION}

To harvest the cooperative gain promised by information theory [1], [2], coding schemes are needed that can approach the fundamental limits of the relay channel. Among the early coding results for the relay channel were distributed coded diversity schemes [3], [4], [5], which proposed convolutional and turbo codes for the fading channel under cooperation. Later on, Duman et al. [6], [7] proposed turbo

This work was presented in part in the 2010 IEEE International Symposium on Information Theory.

Thuy Van Nguyen and Aria Nosratinia are with the Department of Electrical Engineering, The University of Texas at Dallas, Richardson, TX 75083-0688 USA, Email nvanthuy@utdallas.edu, aria@utdallas.edu. Dariush Divsalar is with the Jet Propulsion Laboratory, California Institute of Technology, Pasadena, CA 91109-8099 USA Email: Dariush.Divsalar@jpl.nasa.gov. 

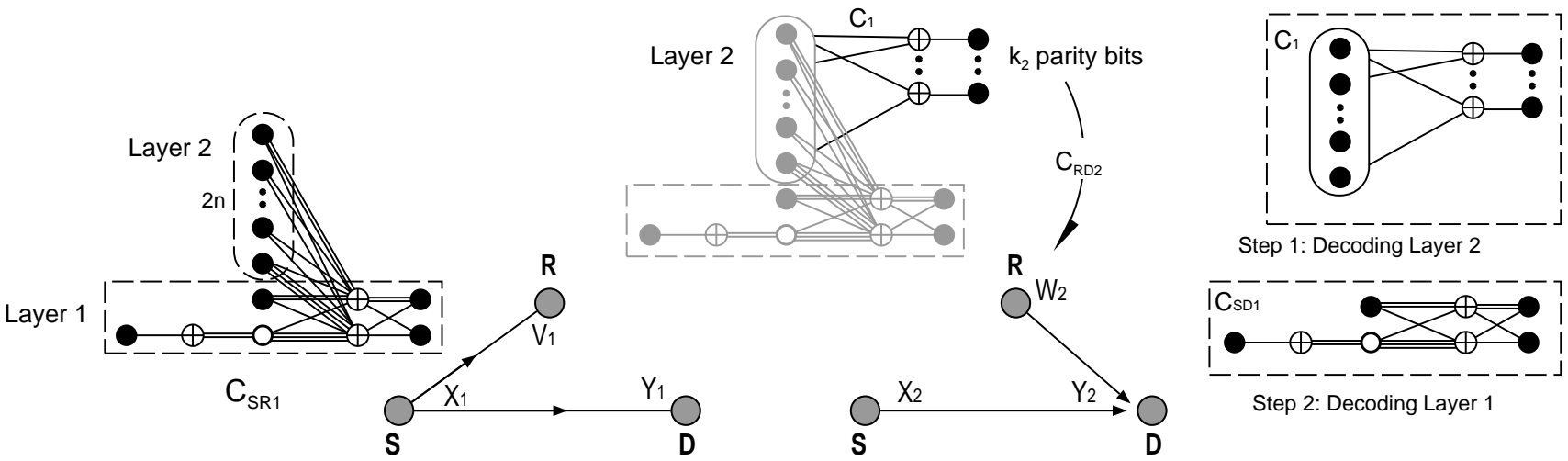

Fig. 1. Bilayer-lengthened protograph design for half-duplex relay with $R_{S D_{1}}=\frac{1}{2}$ and $R_{S R_{1}}=\frac{n+1}{n+2}, n=1,2, \ldots$

codes for the decode-forward (DF) relay channel in half-duplex and full-duplex modes. There is a large body of work focused on designing LDPC codes for the relay channel [8], [9], [10], [11], [12], [13], [14]. These works mostly utilize irregular LDPC codes and use density evolution (or related) techniques to search for optimized irregular LDPC ensembles operating at two different rates. Razaghi and Yu [11] produce bilayer LDPC code structures for the DF scheme. This approach has been refined in other works including [8], [9]. Other examples of LDPC codes for the half-duplex relay channel include [10], [12], [13], [14].

It has been known that the basic problem of coding for the DF relay channel in the power-limited regime can be reduced to the following: a code and its sub-code must be designed simultaneously that operate with two different rates at two different SNRs at the relay and the destination, respectively. Despite some progress, LDPC codes for the relay channel are often painstakingly optimized to match to a set of channel conditions without a structure to facilitate the optimization of design, and many of them do not offer easy encoding. This paper proposes a class of LDPC relay codes that address three important issues in an integrative manner: low encoding complexity, modular structure allowing for easy design, and rate-compatibility so that the code can be easily matched to a variety of channel conditions without extensive re-optimization. In addition, the proposed codes offer excellent performance.

In this paper, we concentrate on the DF protocol. For practical purposes, we further limit ourselves to the half-duplex relaying where the relay cannot transmit and receive at the same time. The main contribution of this paper is a coding scheme for the relay channel using protographs [15] that are built with bilayer expurgated and bilayer-lengthened structures [11]. A protograph code [15] is an LDPC code that can be constructed by extension from a small bipartite graph called a protograph, whose graph 
topology is represented by a so-called proto-matrix. Protograph codes can achieve very good thresholds with low encoder complexity as well as fast decoding [16], [17]. The proposed approach offers flexibility in designing a family of rate-compatible embedded codes for relay channels. These embedded codes allow a coding scheme whose rate can easily be adapted to channel conditions, and are thus suitable for designing multi-relay coding systems. We also introduce a methodology to evaluate the end-to-end error performance of relay coding schemes, and demonstrate the end-to-end performance of our proposed codes.

\section{BACKGROUND}

\section{A. System Model}

A half-duplex single-relay channel is shown in Figure $11 X_{i}$ and $W_{i}$ denote the transmitted signals from the source $(\mathrm{S})$ and the relay (R) while $Y_{i}$ and $V_{i}$ denote the received signals at the destination (D) and relay, respectively. Subscript $i=1$ denotes the broadcast mode which is active for a fraction $t$ of the transmission interval, and subscript $i=2$ denotes the multiple access mode which is active for a fraction $t^{\prime}=1-t$ of the transmission interval. The received signals are:

$$
\begin{aligned}
& V_{1}=h_{S R} X_{1}+N_{R_{1}} \\
& Y_{1}=h_{S D} X_{1}+N_{D_{1}} \\
& Y_{2}=h_{S D} X_{2}+h_{R D} W_{2}+N_{D_{2}},
\end{aligned}
$$

where $h_{S D}$ and $h_{R D}$ are the S-to-D and R-to-D channel coefficients, respectively, $N_{R_{1}}, N_{D_{1}}$ and $N_{D_{2}}$ are the noise samples at the relay and the destination in the first and second time slots, respectively. All noise samples are assumed to be Gaussian with zero mean and unit variance. $P_{S_{1}}=E\left[X_{1}^{2}\right]$ represents the source transmission power in the first time slot (duration $t$ ). Similarly $P_{S_{2}}$ and $P_{R_{2}}$ represent the source and relay transmission powers in the relayed time slot (duration $t^{\prime}=1-t$ ). We also define $S N R_{S R}$, $S N R_{S D}$ and $S N R_{R D}$ as signal-to-noise ratios by the relay and destination during the first time slot, and by the destination during the second time slot, respectively.

\section{B. Coding for Half-Duplex Relay Channels}

In the first time slot, a source sends a high-rate $C_{S R_{1}}$ code to a relay and a destination, using a rate that is decodable at the relay. In the second time slot, the relay transmits additional mutual information to help 
the destination decode, producing an effectively lower-rate $C_{S D_{1}}, 1$ Thus the relay coding problem consists of a simultaneous design of two codes that operate at two different SNRs $S N R_{S R}>S N R_{S D}$, such that one is a subset of the other. This problem has been attempted a number of times by forceful optimization, but a more streamlined approach is now available via bilayer expurgated and bilayer-lengthened LDPC structures [11]. In the bilayer LDPC structure, either the bit nodes or the check nodes are divided into two sets (called layers) and the graph, although still a bipartite graph, is re-drawn with two rows of check nodes sandwiching the bit nodes, or vice versa [11, Fig. 3 and 6]. This provides a convenient way to build and illustrate subcodes, which we use for our purposes. For a more comprehensive treatment and justification of the bilayer structure please see [11], [13].

\section{Protograph Codes}

A protograph [15] is a Tanner graph with a relatively small number of nodes. A protograph code is an LDPC code constructed from a protograph by a copy-and-permute operation, where the protograph is copied $N$ times and the $N$ variable-to-check pairs (edges) corresponding to the same edge type of the original protograph are permuted. Thus the protograph code has the same rate and the same node distribution as the original protograph. Several capacity-approaching protograph code designs have been proposed in [17], [18]. Figure 2 shows a rate-1/2 protograph from [19] consisting of 7 variable nodes and 4 check nodes that are interconnected by 24 edge types. The dark circles represent transmitted variable nodes, the white circle is a punctured node and the circles with a plus sign are parity check nodes. This protograph can be represented by the proto-matrix

$$
H_{1 / 2}=\left(\begin{array}{ccccccc}
1 & 2 & 0 & 0 & 0 & 1 & 0 \\
0 & 3 & 1 & 1 & 1 & 1 & 0 \\
0 & 1 & 2 & 2 & 2 & 1 & 1 \\
0 & 2 & 0 & 0 & 0 & 0 & 2
\end{array}\right),
$$

where the rows and columns represent the check nodes and variable nodes respectively. The entries of the matrix represent the number of parallel edges that connect the variable node and the check node. Using the PEXIT technique [20], the threshold of this code is $0.439 \mathrm{~dB}$, a gap of $0.252 \mathrm{~dB}$ of capacity. The

\footnotetext{
${ }^{1}$ In our formulation the source may also be active in the second time slot, but it will be constrained to synchronously transmit the same signal/codebook as the relay. Thus in this work the distinction between orthogonal and non-orthogonal transmission is only in the received SNR during relay time slots. Since we parameterize our analysis by received SNR without any direct reference to source and relay powers, both orthogonal and (synchronous) non-orthogonal transmissions are subsumed in the following developments.
} 


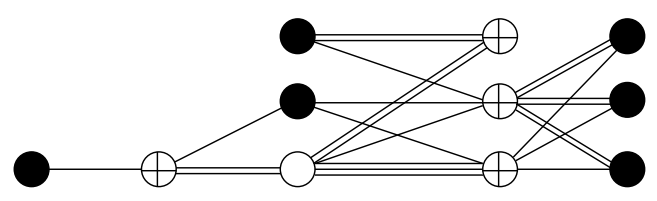

Fig. 2. The rate- $1 / 2$ protograph with a threshold of $0.439 \mathrm{~dB}$

linear minimum distance of this code grows linearly with codelength 2 which is necessary for avoiding an error floor [21].

\section{DESIGN OF BILAYER PROTOGRAPH-BASED LDPC CODES FOR THE RELAY CHANNEL}

\section{A. Bilayer Lengthened Structure}

A bilayer lengthened structure for the relay channel [11] is shown in Fig. 11 where a high-rate code is constructed by adding variable nodes to the graph of a low-rate code. Geometrically, the overall graph contains two layers (sets) of variable nodes and one set of check nodes. One layer of variables connecting to the checks forms a graph of a lower-rate code, and the entire graph constitutes a high-rate code. The corresponding parity check matrix has the form:

$$
H_{S R_{1}}=\left[H_{S D_{1}} H_{e}\right]
$$

where $H_{S D_{1}}$ is the parity matrix of the lower-rate code which is equivalent to the sub-code (Layer 1 or $C_{S D_{1}}$ ) and $H_{e}$ is the extension matrix representing Layer 2. Layers 1 and 2 combined create a capacityapproaching code for the source-relay link $\left(C_{S R_{1}}\right)$ as shown in Fig. 1 The relay decodes this codeword, then protects the codeword bits in Layer 2 via $k_{2}$ parity bits. These parities are encoded with $C_{R D_{2}}$ and transmitted to the destination during the second time slot. The destination, using these $k_{2}$ parity bits, can reliably detect the bits in Layer 2, therefore they can be eliminated from the code graph. The remaining graph contains just Layer 1, which constitutes a capacity approaching code at the SNR of the SD link, which is decodable at the destination.

We will describe in the following an example for the design of the bilayer lengthened codes with rates $R=\frac{n+1}{n+2}, n=1,2 \ldots$, as an extension of the rate-1/2 protograph of Eq. (2). For each new code, three

\footnotetext{
${ }^{2}$ If a random permutation per each edge of its protograph is used.
} 
variable nodes (or columns) are added, as follows:

$$
H_{\frac{n+1}{n+2}}=\left(H_{\frac{n}{n+1}} \mid \begin{array}{ccc}
y_{1} & y_{2} & y_{3} \\
\cline { 2 - 2 } & x_{4} & x_{7} \\
x_{2} & x_{5} & x_{8} \\
x_{3} & x_{6} & x_{9}
\end{array}\right),
$$

where $x_{i}$ and $y_{i}$ variables denote the number of parallel edges in the extension graph, to be determined. Variables $y_{i}$ in the first row correspond to the check node that connects to the degree- 1 variable node. Variables $x_{i}$ in rows 2-4 correspond to the remaining check nodes. In order to preserve the linear growth of minimum distance for the bilayer codes, the column sums in rows $2-4$, namely sums of columns designated with variables $x_{i}$, should be 3 or higher [21].

We further simplify the problem by setting the maximum number of parallel edges to 2 (i.e. $x_{i}, y_{i}=$ $0,1,2)$. These constraints limit the range of parameters thus simplifying the optimization. Our cost function is the threshold which is calculated via the PEXIT method. For this example, the best threshold is given by:

$$
\begin{aligned}
& H_{2 / 3}=\left(H_{1 / 2} \mid \begin{array}{ccc}
0 & 1 & 1 \\
1 & 1 & 1 \\
2 & 1 & 2 \\
0 & 1 & 0
\end{array}\right) H_{3 / 4}=\left(H_{2 / 3} \mid \begin{array}{ccc}
0 & 0 & 2 \\
2 & 2 & 0 \\
1 & 1 & 2 \\
0 & 0 & 1
\end{array}\right) \\
& H_{4 / 5}=\left(H_{3 / 4} \mid \begin{array}{ccc}
0 & 1 & 2 \\
1 & 2 & 2 \\
2 & 1 & 1 \\
0 & 0 & 0
\end{array}\right) H_{5 / 6}=\left(H_{4 / 5} \mid \begin{array}{ccc}
0 & 0 & 1 \\
2 & 2 & 0 \\
1 & 1 & 2 \\
0 & 0 & 2
\end{array}\right) \\
& H_{6 / 7}=\left(H_{5 / 6} \mid \begin{array}{ccc}
0 & 0 & 1 \\
1 & 2 & 1 \\
2 & 1 & 2 \\
0 & 1 & 0
\end{array}\right) H_{7 / 8}=\left(H_{6 / 5} \mid \begin{array}{ccc}
0 & 0 & 2 \\
2 & 2 & 0 \\
1 & 1 & 2 \\
0 & 0 & 2
\end{array}\right) \\
& H_{8 / 9}=\left(H_{7 / 8} \mid \begin{array}{ccc}
0 & 0 & 0 \\
0 & 1 & 2 \\
2 & 2 & 1 \\
1 & 1 & 0
\end{array}\right) \quad H_{9 / 10}=\left(H_{8 / 9} \mid \begin{array}{ccc}
0 & 0 & 2 \\
1 & 2 & 0 \\
2 & 1 & 2 \\
0 & 0 & 2
\end{array}\right)
\end{aligned}
$$


TABLE I

THRESHOLDS OF PROPOSED PROTOGRAPH CODES

\begin{tabular}{|c|c|c|c|}
\hline $\begin{array}{c}\text { Code } \\
\text { Rate }\end{array}$ & $\begin{array}{c}\text { Protograph } \\
\text { threshold (dB) }\end{array}$ & $\begin{array}{c}\text { Capacity } \\
\text { threshold }(\mathrm{dB})\end{array}$ & $\begin{array}{c}\text { Gap to } \\
\text { capacity }\end{array}$ \\
\hline \multicolumn{4}{|c|}{ Bilayer lengthened design } \\
\hline $1 / 2$ & 0.439 & 0.187 & 0.252 \\
$2 / 3$ & 1.223 & 1.059 & 0.164 \\
$3 / 4$ & 1.720 & 1.626 & 0.094 \\
$4 / 5$ & 2.136 & 2.040 & 0.096 \\
$5 / 6$ & 2.455 & 2.362 & 0.093 \\
$6 / 7$ & 2.718 & 2.625 & 0.093 \\
$7 / 8$ & 2.941 & 2.845 & 0.099 \\
$8 / 9$ & 3.125 & 3.042 & 0.083 \\
$9 / 10$ & 3.295 & 3.199 & 0.096 \\
\hline \multicolumn{5}{|c|}{ Bilayer expurgated design } \\
\hline $3 / 4$ & 1.720 & 1.626 & 0.094 \\
$2 / 3$ & 1.182 & 1.059 & 0.123 \\
$7 / 12$ & 0.809 & 0.590 & 0.219 \\
$1 / 2$ & 0.420 & 0.187 & 0.233 \\
$5 / 12$ & 0.144 & -0.185 & 0.329 \\
$1 / 3$ & -0.263 & -0.497 & 0.234 \\
\hline
\end{tabular}

The iterative decoding thresholds of these codes calculated by PEXIT technique [20] are given in Table I. For rates $>2 / 3$, the produced codes have iterative decoding thresholds within $0.1 \mathrm{~dB}$ of the capacity, and the rate-2/3 code has a threshold within $0.152 \mathrm{~dB}$.

\section{B. Design of Bilayer Expurgated Protograph Codes}

A bilayer expurgated structure for the relay channel [11] is shown in Fig. 3 where a low-rate code is constructed by adding check nodes to the graph of a high-rate code. The bilayer graph in this case contains two layers (sets) of check nodes and one set of variable nodes (unlike the bilayer lengthened structure). The variable nodes together with one layer of check nodes form the graph of a high-rate code, and the entire graph constitutes a low-rate code. The corresponding parity check matrix has the form

$$
H_{S D_{1}}=\left[\begin{array}{c}
H_{S R_{1}} \\
H_{e}
\end{array}\right] \text {, }
$$




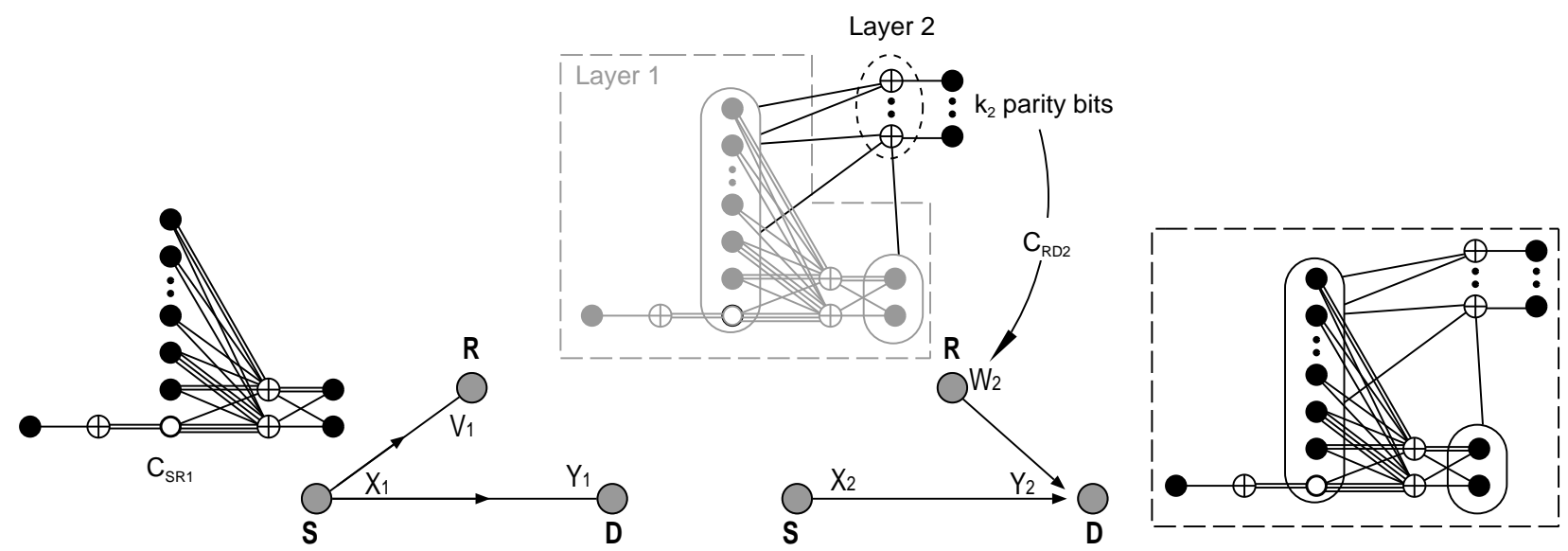

Fig. 3. Bilayer expurgated protograph design for half-duplex relay with $R_{S D_{1}}=\frac{N-k_{1}-k_{2}}{N}$ and $R_{S R_{1}}=\frac{N-k_{1}}{N}, N$ is the number of variable nodes

where $H_{S R_{1}}$ is the parity check matrix of a high-rate LDPC capacity-approaching code for the sourcerelay link (representing the sub-code $C_{S R_{1}}$ of Layer 1), and $H_{e}$ is the extension matrix representing Layer 2. Layers 1 and 2 together create a capacity-approaching code $C_{S D_{1}}$ for the source-destination link as shown in Fig. 3 . The source transmits a $C_{S R_{1}}$ codeword. The relay, after decoding the source codeword, produces $k_{2}$ additional "parity" bits using the extension matrix $H_{e}$, re-encodes these $k_{2}$ bits with a codebook $C_{R D_{2}}$ and transmits to the destination. At the destination, the $k_{2}$ parity bits that are reliably detected essentially provide $k_{2}$ additional check values on the source codeword from $C_{S R_{1}}$. This is equivalent to decoding a $C_{S D_{1}}$ codeword (with a lower rate) at the SNR of the source-destination link.

We now describe an example for the design of bilayer expurgated codes. In the interest of brevity, we only present one construction of bilayer codes. However, the proposed method is completely general; we can start from any high-rate code to build a family of expurgated codes. The starting point for the following construction is the rate-3/4 protograph code designed in the last subsection. The rate-3/4 protograph contains 13 variable nodes (one of them punctured) and 4 check nodes. Low-rate bilayer codes expurgated from this code have rates $R=\frac{13-4-n}{13-1}$, where $n$ is the number of checks added. For each value of $n$, the new code will require a search. As a representative sample, we concentrate on the search for $n=1$, which yields a rate- $2 / 3$ code. The search space for this new code is in the form 


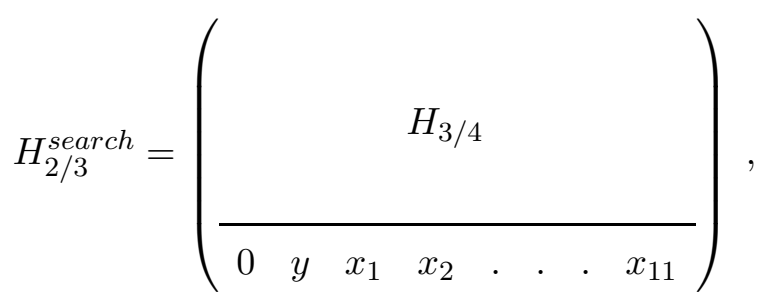

where $y$ variable denotes the number of parallel edges connecting to the punctured variable node and $x_{i}$ variables denote the number of parallel edges connecting to remaining variable nodes except the degree-1 variable node. Having a degree- 1 variable node improves the iterative decoding threshold of the protograph code [17].3

The optimization process is simplified by further limiting the maximum number of parallel edges. For example, we can limit $y \in\{1,2\}$ and $x_{i} \in\{0,1,2\}$. Proto-matrices of new expurgated protographs are given by

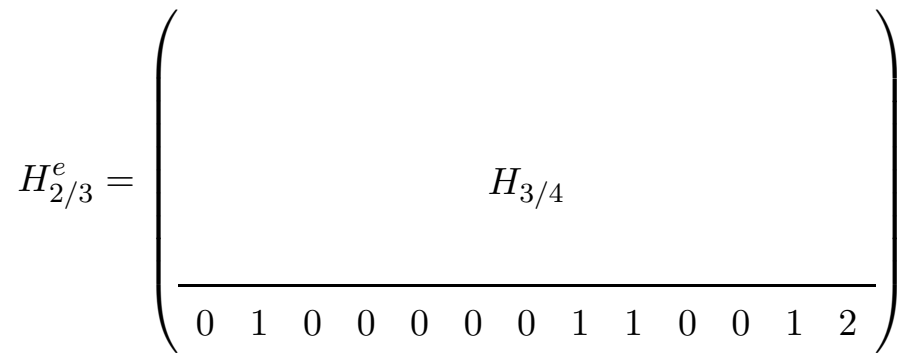

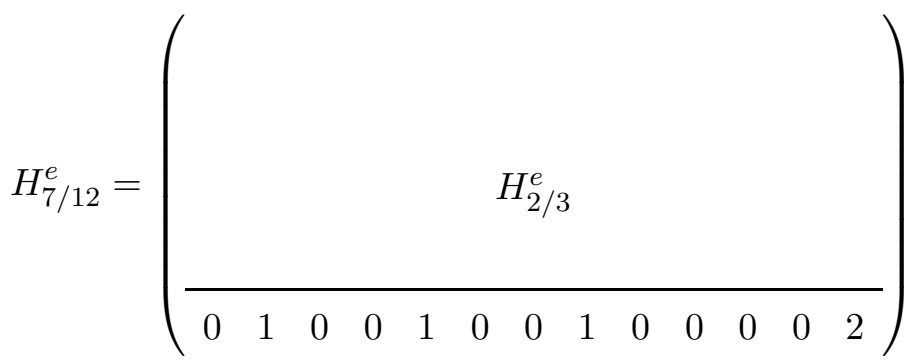

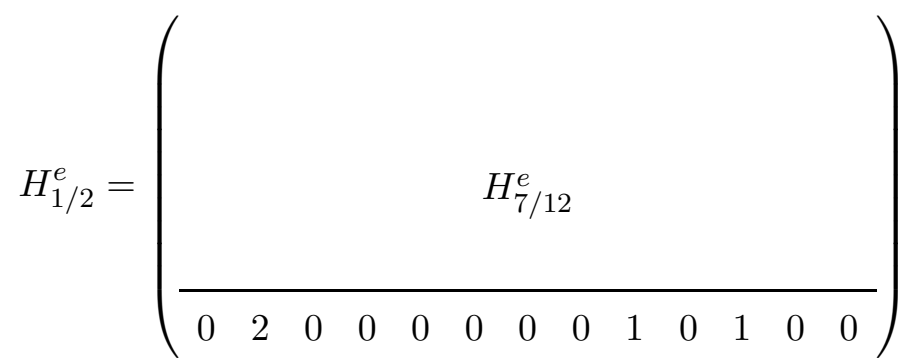

${ }^{3}$ It is well known that degree-1 nodes are not to be used in irregular LDPC codes, but their effectiveness in structured LDPC codes is well documented [22]. 


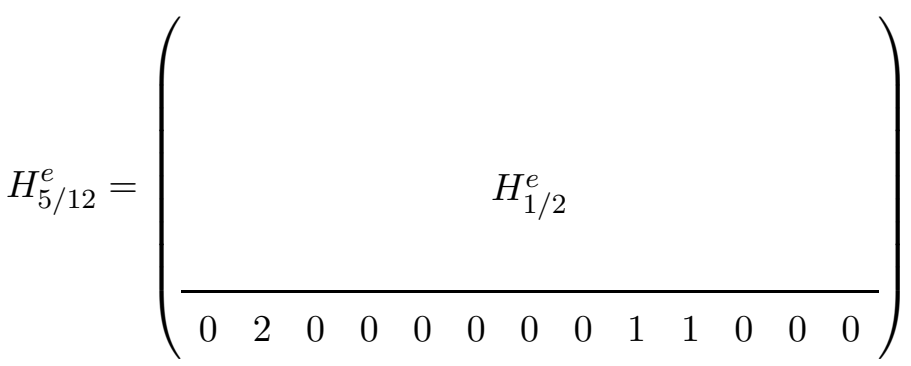

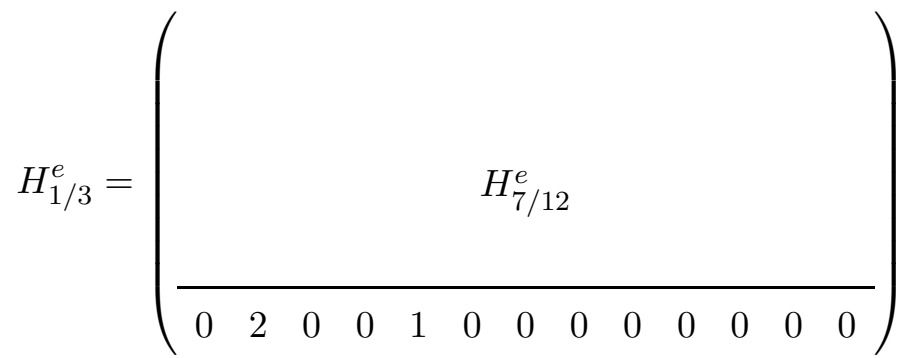

The iterative decoding thresholds of these codes are given in Table I] The produced codes all have decoding thresholds within $0.23 \mathrm{~dB}$ of the capacity except the rate-7/12 one which has a decoding threshold within $0.33 \mathrm{~dB}$ of the capacity.

\section{NUMERICAL RESULTS}

\section{A. Coding thresholds}

We design nested bilayer lengthened and expurgated protograph codes for the half-duplex relay channel, which can operate at any two rates with iterative decoding thresholds close to capacity, as shown in Table I] For comparison, the thresholds of bilayer lengthened and bilayer expurgated irregular LDPC codes proposed in the literature are shown in Table $\amalg$ All codes use a rate pair $\left(R_{S D_{1}} \simeq 0.5, R_{S R_{1}} \simeq 0.7\right)$. Our proposed bilayer lengthened and bilayer expurgated codes are better than the codes proposed in [14] and within $0.1 \mathrm{~dB}$ of codes reported in other works 4 . Thus, a simple design approach has yielded a family of codes that offer a performance comparable with other highly-optimized bilayer LDPC codes, while offering important advantages in terms of rate-compatibility, low encoding complexity, and the fast decoding properties of protograph codes.

\section{B. Simulation Results}

So far we have represented codes only in the form of proto-matrices (protographs). As mentioned in Section $\amalg-C$, a protograph code (an equivalent LDPC code) is a large derived graph constructed by copy-and-permutation operation on protograph, a process known as lifting.

\footnotetext{
${ }^{4}$ The rate of [11], [14] is slightly less than $1 / 2$, thus slightly skewing the comparison in their favor.
} 
TABLE II

COMPARISON OF THRESHOLDS $\left(R_{1} \simeq 0.5, R_{2} \simeq 0.7\right)$

\begin{tabular}{|l|c|c|c|c|c|c|c|c|c|}
\hline & \multicolumn{4}{|c|}{ Lengthened } & \multicolumn{5}{c|}{ Expurgated } \\
\cline { 2 - 10 } & {$[11]$} & {$[14]$} & {$[8]$} & Our BL code & {$[11]$} & {$[9]$} & {$[8]$} & {$[14]$} & Our BE code \\
\hline Gap $_{1}$ & 0.164 & 0.3854 & 0.1039 & 0.239 & 0.514 & 0.258 & 0.284 & 0.6323 & 0.233 \\
\hline Gap $_{2}$ & 0.120 & 0.1758 & 0.0945 & 0.152 & 0.084 & 0.084 & 0.084 & 0.215 & 0.123 \\
\hline
\end{tabular}

Our protograph codes are derived from protographs in two lifting steps. First, the protograph is lifted by a factor of 4 using the progressive edge growth (PEG) algorithm [23] in order to remove all parallel edges. Then, a second lifting using the PEG algorithm was performed to determine a circulant permutation of each edge class that would yield the desired code block length.

In our nested bilayer lengthened codes of Section $\amalg$ II-A the parity check matrix for the lower-rate code can be obtained by removing certain columns from the parity check matrix of the higher rate code, and this produces economies in the design of the decoders. In fact, it is enough to design a decoder for the largest rate code (9/10). To decode the lower-rate codes, the missing coded bits are replaced by erasures at the decoder. In the same manner, the bilayer expurgated codes generated in Section $\amalg$ II-B only need the decoder of the lowest rates $(1 / 3)$. Other higher-rate codes are decoded by ignoring redundant checks at the common decoder.

The performances of our bilayer lengthened protograph codes with rates $1 / 2,2 / 3$ and $3 / 4$ over a binary-input additive white Gaussian noise (BI-AWGN) are shown in Fig. 4, In addition, performances of bilayer expurgated protographs with rates $1 / 3,5 / 12,1 / 2,2 / 3$ and $3 / 4$ are shown in Fig. 5 . All codes are simulated with the information block-length of $16 \mathrm{k}$. To construct codes with this $16 \mathrm{k}$ information block-length, the protographs for the lengthened codes are first lifted by a factor of 4 , and subsequently again by factors 1365,683 , and 455 , for codes of rate $1 / 2,2 / 3$, and $3 / 4$, respectively. A similar two-step lifting is used for the expurgated codes.

The decoder is a standard message passing decoder where the maximum number of iterations is set to 200. Log-likelihood ratio (LLR) clipping and other decoding parameters are according to [24]. No error floors were observed down to the word error rate (WER) $10^{-6}$. The gap to capacity of these codes is within $0.6 \mathrm{~dB}$ of their iterative decoding threshold and within $0.8 \mathrm{~dB}$ of their capacity limits at $W E R \approx 3 \times 10^{-6}$.

We now consider a half-duplex relay channel with information block-length of 16380 bits. We assume 


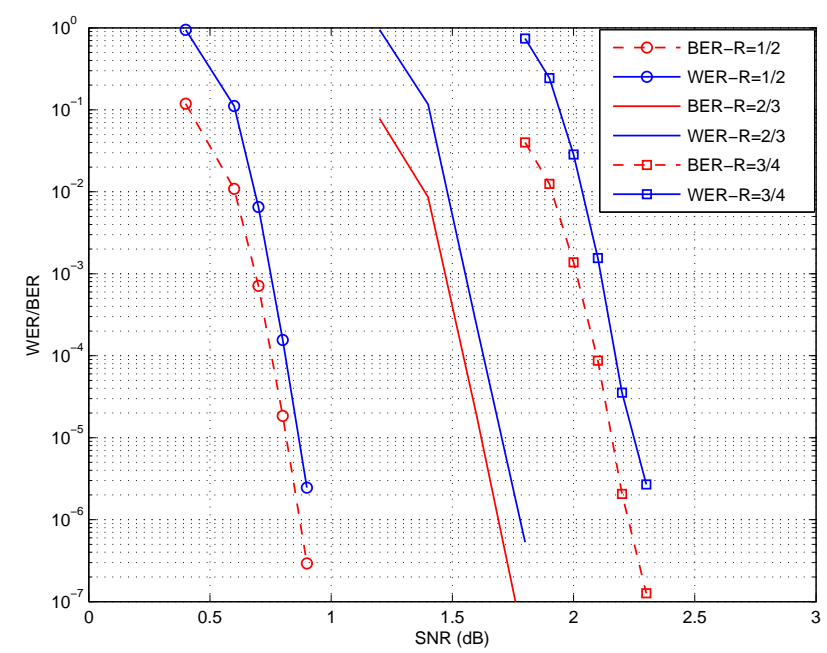

Fig. 4. Performance of rate $1 / 2,2 / 3,3 / 4$ bilayer lengthened protograph codes designed in Section $\amalg$ II-A with information blocklength of $16 \mathrm{k}$

the SR link supports rate $R_{S R_{1}}=3 / 4$ and the SD link supports rate $R_{S D_{1}}=1 / 2$. For this example, the time division $t=0.75$ is chosen for the source and relay. The rate needed in the RD link is $R_{R D_{2}}=3 / 4$ [13, Eq. 5]. This is the same as the rate of SR code but with a different codeword-length. Under these conditions, the achievable rate of the relay channel is 0.5625 [13, Eq. 3].

We now consider the bilayer expurgated coding scheme (Fig. 3) with information blocklength 16380. $C_{S R_{1}}$ is implemented by the rate-3/4 protograph given in Section $\amalg$ II-A. The code $C_{R D_{2}}$, which protects additional parities generated at the relay, has information blocklength $5460 . C_{R D_{2}}$ is constructed from the same protograph as $C_{S R_{1}}$, but with a shorter length. The destination decodes a $C_{S R_{1}}$ codeword plus these additional parity values, which constitutes a codeword of $C_{S D_{1}}$ in the same manner as [11]. The word error rate (WER) and bit error rate (BER) performances of these codes are shown in Fig. 6, The shorter block length of the rate-3/4 RD code has resulted in a wider waterfall region, highlighting a weakness of the successive decoding approach at smaller block lengths.

The bilayer lengthened structure involves four codes (Fig. 1). Using the above relay channel parameters, $C_{S R_{1}}$ has the information block-length 16380 and codeword block length 21840 . Each codeword of $C_{S R_{1}}$ is composed of a $C_{S D_{1}}$ codeword with blocklength 10920 and an extension of 10920 bits derived via the extension matrix $H_{e}$ from Eq. (3). The relay, after decoding and recovering the codeword, multiplies the extension bits by the parity check matrix of a rate- $1 / 2$ code $C_{1}$ to calculate a syndrome of length 


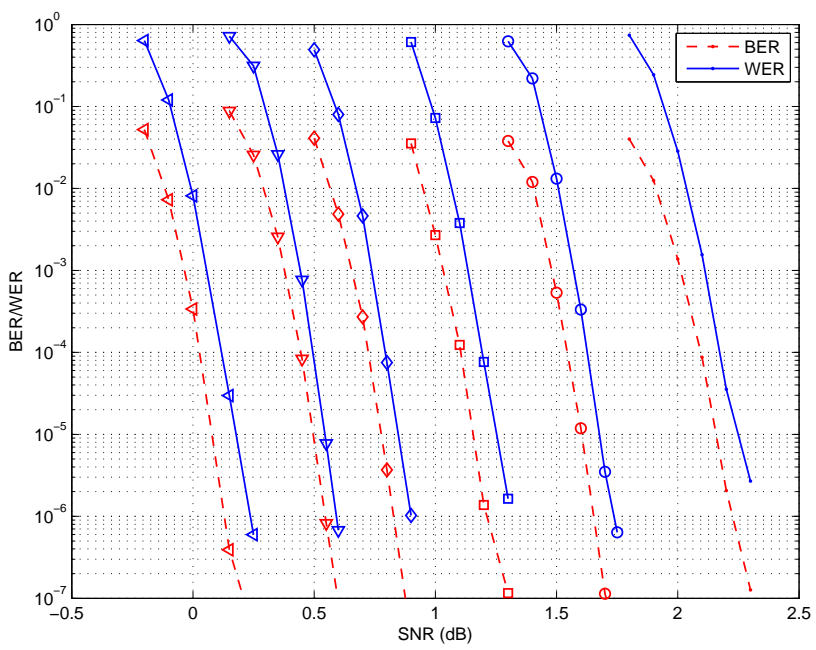

Fig. 5. Performance of rate $1 / 3,5 / 12,1 / 2,7 / 12,2 / 3,3 / 4$ bilayer expurgated protograph codes (Section $\Pi$ I-B), information block-length $16 \mathrm{k}$

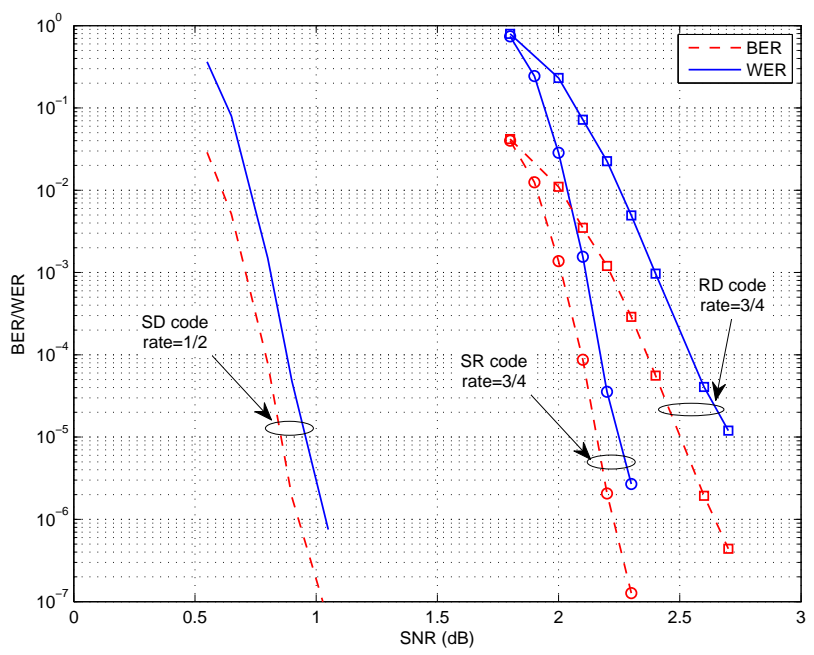

Fig. 6. Performance of component codes for a relay channel: $R_{S D_{1}}=1 / 2, R_{S R_{1}}=3 / 4$ and $R_{R D_{2}}=3 / 4$ using bilayer expurgated structure (Section $\Pi$ II-B) with $\alpha=1.4 \mathrm{~dB}$ and $\beta=1.6 \mathrm{~dB}$ 


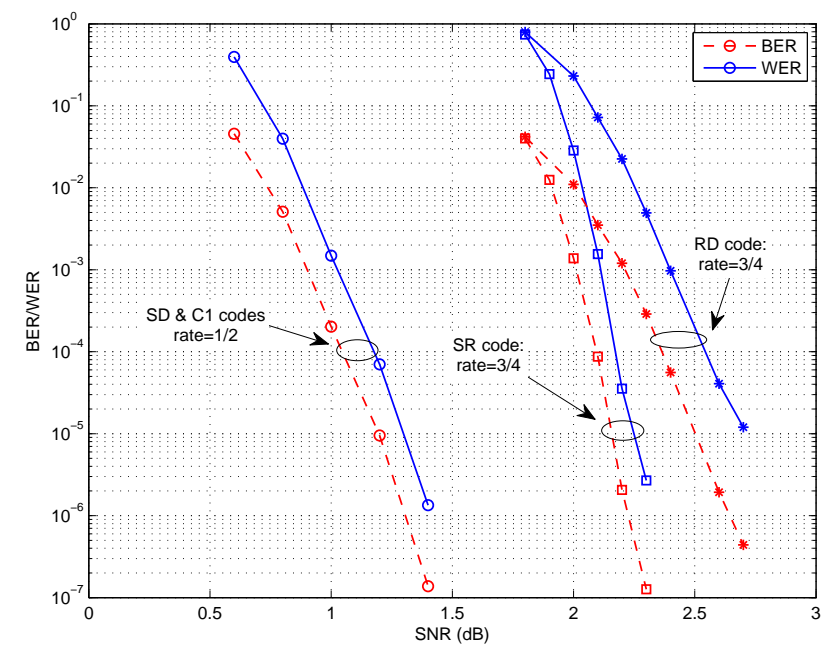

Fig. 7. Performance of component codes for a relay channel: $R_{S D_{1}}=1 / 2, R_{S R_{1}}=3 / 4, R_{C_{1}}=1 / 2$ and $R_{R D_{2}}=3 / 4$ using bilayer lengthened structure in Section $\amalg$ - with $\alpha=1.4 \mathrm{~dB}$ and $\beta=1.6 \mathrm{~dB}$

5460. $C_{1}$ is lifted from the rate-1/2 protograph given in Eq. (2). The syndrome is then encoded by $C_{R D_{2}}$, transmitted and decoded at the destination to recover the syndrome. $C_{S R_{1}}$ and $C_{R D_{2}}$ are similar to their counterparts in the bilayer expurgated scheme. Also, $C_{S D_{1}}$ and $C_{1}$ have the same code structure and blocklength. The WER and BER performances are shown in Fig. 7 Performance of $C_{S D_{1}}$ in the bilayerlengthening structure is worse than in the bilayer expurgated structure due to its shorter blocklength.

Although the example above was coached in terms of orthogonal transmissions, it also applies to a non-orthogonal system with correspondingly lower source and relay powers (see footnote 1).

\section{End-to-End Performance of Relay Coding Systems}

Many works in contemporary literature represent the performance of relay channel codes by illustrating two component codes: the source-relay code, and the source-destination code. In this approach it is implicitly assumed the relay-destination code is an ideal code. However, the relay-destination code often operates at a smaller block length, thus it has a wider waterfall and may very well be the bottle neck for the entire system, therefore providing the thresholds or even simulations for only the two other subcodes may not always be fully illuminating of the overall performance. We believe there is need for a comprehensive end-to-end performance metric.

In general the end-to-end relay channel error is a function of the three SNRs of its constituent channels. But showing this dependence requires a four-dimensional plot, which is not practical. We construct a 
simple but useful error plot by assuming

$$
S N R_{S R}=S N R_{S D}+\alpha \quad S N R_{R D}=S N R_{S D}+\beta
$$

where $\alpha$ and $\beta$ are fixed constants. The affine relationship of the SNRs allows them to be displayed on the same axis, producing a simple end-to-end error plot which can be thought of as a 2-dimensional slice of the general four-dimensional error characterization of the relay channel. $\alpha$ and $\beta$ can be carefully chosen (as shown below) to generate a reasonable and insightful overall representation of the performance of the relay channel that does not ignore or trivialize any of the parameters of the system.

To simplify the simulations, we develop a tight bound on the end-to-end error based on component errors in the system. For the purposes of exposition we concentrate on the expurgated-bilayer codes (Figure 3), where three types of errors may happen in the system: $E_{R}$ is the error event at the relay, $E_{R D}$ is the error in the decoding of the "extra parity" arriving from the relay to the destination, and finally, $E_{D}$ is the error event at the destination in the final decoding of the source message, and the complement of event $E$ is shown with $\bar{E}$. The bound is shown in Eq. (10).

$$
\begin{aligned}
P_{e} & =P\left(E_{D} \mid \overline{E_{R}}\right) P\left(\overline{E_{R}}\right)+P\left(E_{D} \mid E_{R}\right) P\left(E_{R}\right) \\
& =\left[P\left(E_{D} \mid \overline{E_{R D}}, \overline{E_{R}}\right) P\left(\overline{E_{R D}}\right)+P\left(E_{D} \mid E_{R D}, \overline{E_{R}}\right) P\left(E_{R D}\right)\right] P\left(\overline{E_{R}}\right)+P\left(E_{D} \mid E_{R}\right) P\left(E_{R}\right) \\
& \leq P\left(E_{D} \mid \overline{E_{R D}}, \overline{E_{R}}\right)+P\left(E_{R D}\right)+P\left(E_{R}\right)
\end{aligned}
$$

It is easy to see the bound is tight because component codes are used in a regime where their errors are, conservatively, no more than $10^{-2} \sim 10^{-3}$, therefore with a very good approximation $P\left(\overline{E_{R}}\right) \approx 1$, $P\left(\overline{E_{R D}}\right) \approx 1, P\left(E_{D} \mid E_{R}\right) \approx 1$ and $P\left(E_{D} \mid E_{R D}, \bar{E}_{R}\right) \approx 1$.

We are now ready to calculate the values of $\alpha$ and $\beta$, which will determine how the individual code error characteristics are combined to produce the end-to-end performance. We are interested in cases where none of the component errors dominate the others, because when one of the link errors dominates, the performance will be essentially determined by the code on the dominating link, which is already known from the literature on point-to-point channels. So the interesting case is when no link errors dominate, i.e. the waterfall regions of the component curves coincide at their starting points (approximately the threshold).

The performance of two bilayer codes with a rate pair $\left(R_{S D}=1 / 2, R_{S R}=3 / 4\right), \alpha=1.4 \mathrm{~dB}, \beta=1.6$ $\mathrm{dB}$ and time sharing $t=0.75$ are shown in Fig. 8 . To understand how far is the end-to-end performance of our codes from theoretical limits, we follow the convention of point-to-point channels and produce a value of SNR at which a random code of infinite length and rate similar to the code under study is 


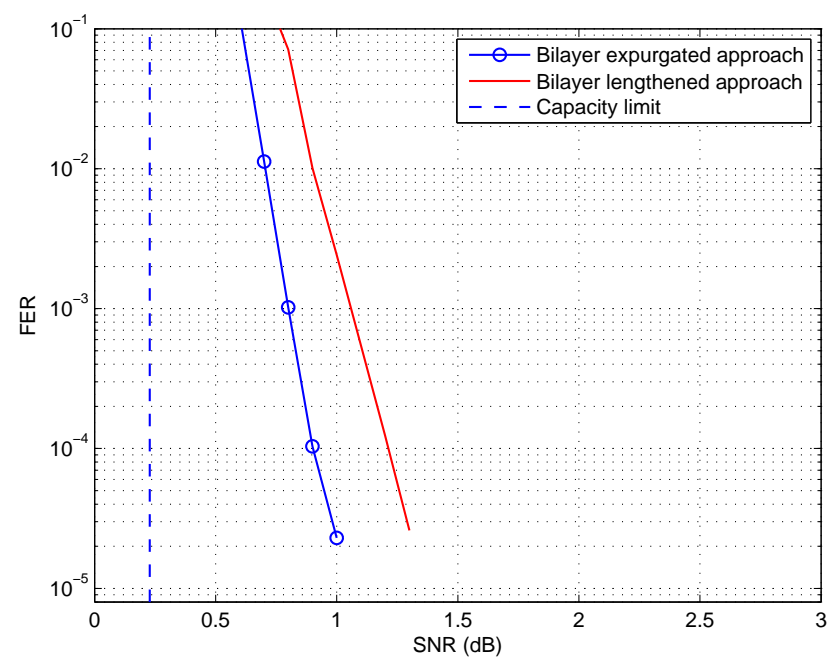

Fig. 8. End-to-end performance bounds for relay coding schemes in Fig. 7 and Fig. 6 with $\alpha=1.4 \mathrm{~dB}$ and $\beta=1.6 \mathrm{~dB}$

capable of theoretically supporting error-free communication. Similarly to the point-to-point case, this involves inverting the capacity formula by inserting the rate and extracting the corresponding SNR. To produce a single SNR value, we use Eq. (7), assume that each of the component codes is at the rate that the mutual information of the link supports, and use the relay mutual information formulas in [13]. Using these parameters, the relay channel of the above example has a throughput of 0.5625 and the code rates correspond to the limiting SNR of $S N R_{S D}=0.225 \mathrm{~dB}$. As seen in this figure, the gap-to-capacity of bilayer expurgated and bilayer lengthened relay coding schemes are about $0.7 \mathrm{~dB}$ and $1.2 \mathrm{~dB}$ respectively at the frame error rate (FER) of $2 \times 10^{-5}$. The end-to-end error bound of the bilayer lengthened code is worse than that of the bilayer expurgated code because of the block-length issues. This phenomenon has also been reported by several previous works including [11], [14].

\section{Desing of Bilayer Codes for Two-Relay Channels}

In a practical two-relay scenario (Fig. 9) it is likely that the relays do not have precisely and deterministically identical channels to the source, destination, and each other. Therefore one relay is likely to be "stronger" and decode first, then this relay will be able to help another relay to decode, and then the two relays together will assist the destination.

We consider transmissions in three time slots for the source and two relays. The transmitted signals from the source (S) and two relays $\left(R_{1}\right.$ and $\left.R_{2}\right)$ are denoted with $X, W$ and $Q$, respectively, and the received signals at the destination and two relays are denoted with $Y_{i}, V_{i}$ and $P_{i}$, respectively, where $i$ 

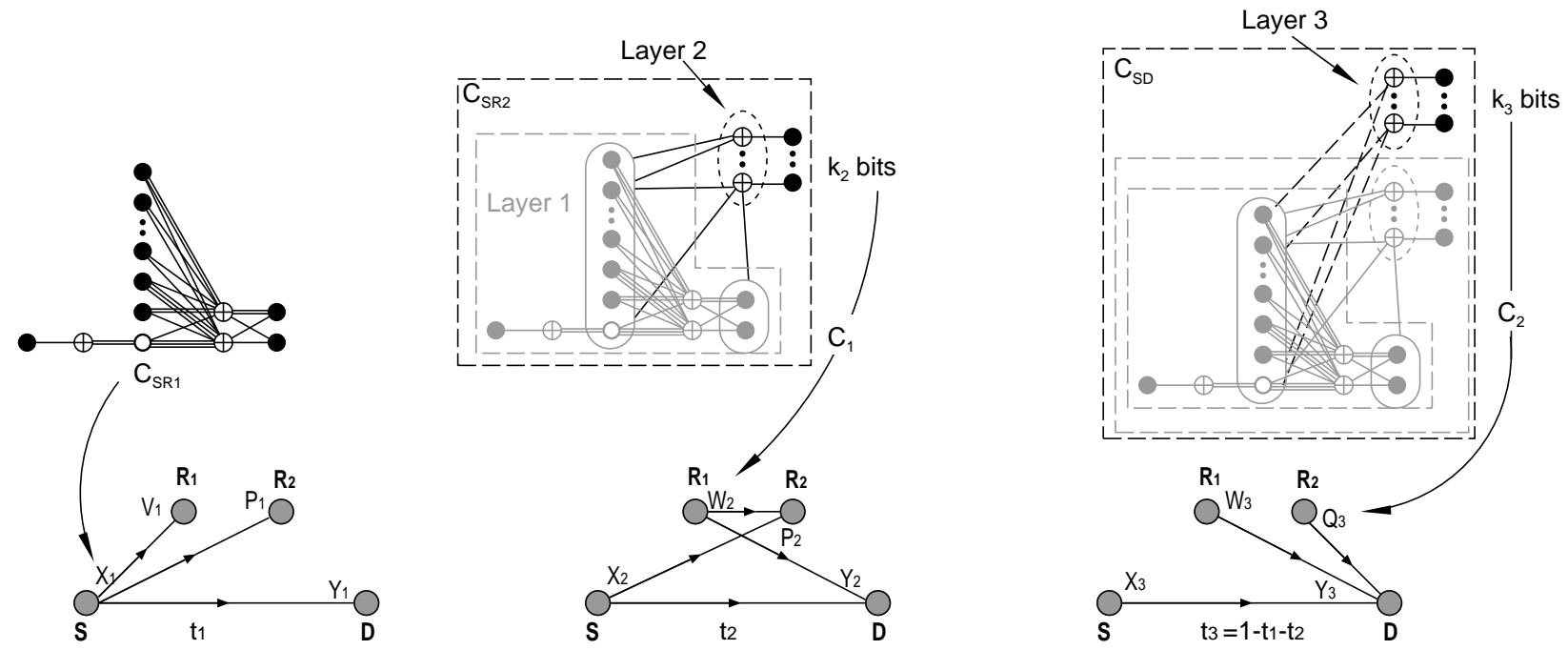

Fig. 9. The expurgated coding structure for the half-duplex two-relay channel

indicates the time slot index. In the first time slot, the source encodes its message using the code $C_{S R_{1}}$. The first relay decodes the source message, but the second relay and the destination cannot (yet). During the second time slot, the first relay generates $k_{2}$ parity bits using the sub-graph denoted Layer $2 \sqrt[5]{5}$ then encodes these parity bits using another LDPC code $C_{1}$. The second relay, after decoding the $k_{2}$ parity bits, decodes the source message. Then, the second relay computes $k_{3}$ parity bits using the subgraph denoted Layer 3 , encodes the $k_{3}$ bits with another LDPC code $C_{2}$, and transmits to destination. The destination decodes $C_{1}, C_{2}$, and finally the source message with the help of $k_{2}+k_{3}$ additional parity bits from two relays. The achievable rate using the above strategy is a special case of the achievable rate in [25].

For demonstration, consider an example where $R_{1}, R_{2}$ and $D$ can reliably receive source signals with a rate $R_{S R_{1}}=3 / 4, R_{S R_{2}}=7 / 12$ and $R_{S D}=1 / 3$, respectively. The $R_{1}$-to- $D$ channel supports $R_{R_{1} D}=1 / 2$. Assuming $t_{1}=0.6, t_{2}=0.2$ and $t_{3}=0.2$, the achievable rate of the two-relay channel is 0.45 [13]. The rate of $C_{1}$ and $C_{2}$ are $1 / 2$ and 3/4, respectively. Information block length is $16380 . C_{S R_{1}}$, $C_{S R_{2}}$ and $C_{S D}$ are constructed from protographs given in Section $\amalg$ III-B $C_{S R 2}$ is rate-7/12 and $C_{S D}$ is rate- $1 / 3$, resulting in $k_{2}=3640$ and $k_{3}=5460$. Although the explanation of this example was in terms of orthogonal transmissions, the precise same example also applies to a non-orthogonal (beamforming) relay channel with correspondingly lower source and relay power.

${ }^{5}$ Like the one-relay case, these bits are the syndrome of a parity check extension matrix. 


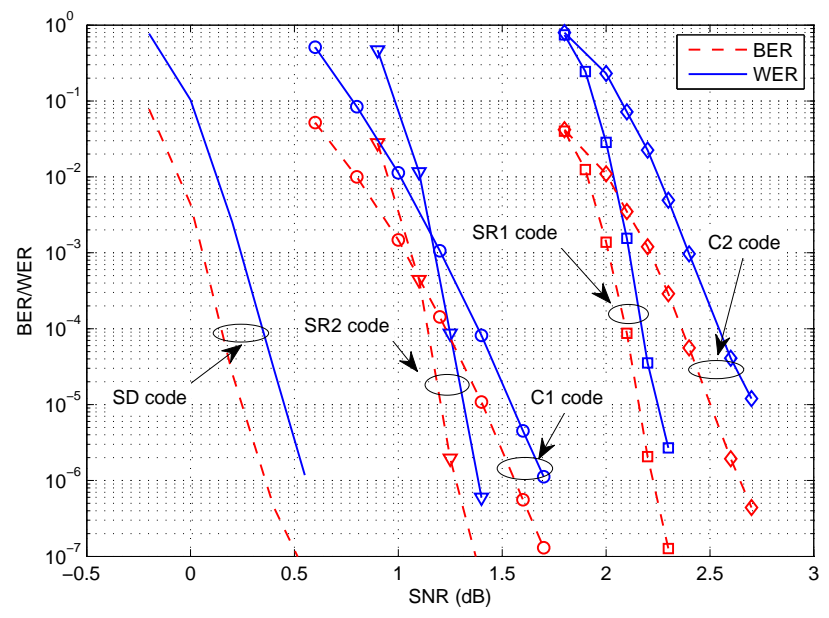

Fig. 10. Performances of component codes used in a two-relay channel: $R_{S R_{1}}=3 / 4, R_{S R_{2}}=7 / 12, R_{S D}=1 / 3, R_{C_{1}}=1 / 2$ and $R_{C_{2}}=3 / 4$. $C_{S R_{1}}, C_{S R_{2}}$ and $C_{S D}$ all have codeword blocklengths of 23660 , while $C_{1}$ and $C_{2}$ have codeword blocklengths of 7280 .

$C_{1}$ arises from the protograph in Eq. (2) and $C_{2}$ is the same code as $C_{S R_{1}}$, but with a shorter blocklength. Fig. 10 shows the performance of $C_{S R_{1}}, C_{S R_{2}}, C_{S D}, C_{1}$ and $C_{2}$, which operate within 0.6 $\mathrm{dB}, 0.7 \mathrm{~dB}, 0.9 \mathrm{~dB}, 1.5 \mathrm{~dB}$ and $1 \mathrm{~dB}$, respectively of their capacity limits at $W E R=10^{-5}$. As expected, $C_{1}$ is the bottleneck in this two-relay channel because of its short blocklength. We note that this gap is an outcome of a relatively short overall block length and a successive decoding approach, both of which are practical considerations and can be relaxed.

Remark 1: In the multi-relay scenario, there are multiple options for the decoding and transmission at relays. For example, two relays can cooperatively beamform towards a destination. The required codes in this case are fundamentally similar to the one-relay channel, therefore we do not consider them separately in this paper.

Remark 2: In the multi-relay scenario the transmission time is divided multiple times which shortens the component block-lengths. Therefore we did not pursue a multi-relay generalization of the bilayer lengthened structure due to its susceptibility to short block length effects.

Remark 3: Optimization of the time division to maximize the achievable rates has been pursued in papers on the capacity of relay channel [26] and can be extended to the multi-relay scenario. Depending on the channel gains, the optimal length for one of the time slots may be zero, in which case one of the relays must be shut off. 
Remark 4: The developments throughout this paper apply to either orthogonal relaying or to nonorthogonal relaying where concurrent transmissions use the same codebook.

\section{Discussion AND CONCLUSION}

This paper presents a simple approach for constructing relay coding schemes based on bilayer lengthened and bilayer expurgated protograph codes which perform within a fraction of $\mathrm{dB}$ of the capacity. The proposed codes allow easy design, flexibility in matching to various relay channel conditions and low encoding complexity and can be extended to multi-relay networks. A framework for end-to-end performance evaluation of the relay codes is also provided.

Nested protograph codes have also been used for the point-to-point channel [27] where, like the present work, multi-component protograph codes have been considered. However, in relay channels the component codes are emitted by different transmitters, unlike the point-to-point case, and see different channels with different SNRs. Thus, relay networks represent a different scenario whose challenges are addressed by a bilayer structure [11] for the overall code and a coset code at the relay, techniques that have no counterpart in the point-to-point case.

\section{ACKNOWLEDGMENT}

This research was supported in part by the VOSP program from the Ministry of Education and Training, Vietnam, and in part by the grant 009741-0084-2007 from THECB. This research was in part carried out at the Jet Propulsion Laboratory, California Institute of Technology, under a contract with NASA.

\section{REFERENCES}

[1] T. Cover and A. E. Gamal, "Capacity theorems for the relay channel," IEEE Trans. Inform. Theory, vol. 25, no. 5, pp. 572-584, Sept. 1979.

[2] G. Kramer, M. Gastpar, and P. Gupta, "Cooperative strategies and capacity theorems for relay networks," IEEE Trans. Inform. Theory, vol. 51, no. 9, pp. 3037 - 3063, Sept. 2005.

[3] M. Janani, A. Hedayat, T. Hunter, and A. Nosratinia, "Coded cooperation in wireless communications: space-time transmission and iterative decoding," IEEE Trans. Signal Processing, vol. 52, no. 2, pp. 362 - 371, Feb. 2004.

[4] T. Hunter and A. Nosratinia, "Cooperation diversity through coding," in Proc. IEEE ISIT, June 2002, p. 220.

[5] M. Valenti and B. Zhao, "Distributed turbo codes: towards the capacity of the relay channel," in Proc. IEEE Vehicular Technology Conference (VTC), vol. 1, Oct. 2003, pp. 322 - 326.

[6] Z. Zhang and T. Duman, "Capacity approaching turbo coding for half duplex relaying," in Proc. IEEE ISIT, Sept. 2005, pp. $1888-1892$.

[7] — - "Capacity-approaching turbo coding and iterative decoding for relay channels," IEEE Trans. Commun., vol. 53, no. 11 , pp. 1895 - 1905, Nov. 2005. 
[8] M. Azmi, J. Yuan, J. Ning, and H. Q. Huynh, "Improved bilayer LDPC codes using irregular check node degree distribution," in Proc. IEEE ISIT, July 2008, pp. 141-145.

[9] M. Azmi and J. Yuan, "Design of multi-edge-type bilayer-expurgated LDPC codes," in Proc. IEEE ISIT, July 2009, pp. 1988-1992.

[10] J. Hu and T. M. Duman, "Low density parity check codes over half-duplex relay channels," in Proc. IEEE ISIT, July 2006, pp. $972-976$.

[11] P. Razaghi and W. Yu, "Bilayer low-density parity-check codes for decode-and-forward in relay channels," IEEE Trans. Inform. Theory, vol. 53, no. 10, pp. 3723-3739, Oct. 2007.

[12] C. Li, G. Yue, X. Wang, and M. A. Khojastepour, "LDPC code design for half-duplex cooperative relay," IEEE Trans. Wireless Commun., vol. 7, no. 11, pp. 4558-4567, Nov. 2008.

[13] A. Chakrabarti, A. D. Baynast, A. Sabharwal, and B. Aazhang, "Low density parity check codes for the relay channel," IEEE J. Select. Areas Commun., vol. 25, no. 2, pp. 280-291, Feb. 2007.

[14] J. P. Cances and V. Meghdadi, "Optimized low density parity check codes designs for half duplex relay channels," IEEE Trans. Wireless Commun., vol. 8, no. 7, pp. 3390-3395, July 2009.

[15] J. Thorpe, “Low-density parity-check (LDPC) codes constructed from protogrtaphs," IPN Progress Report 42-154, Aug. 2003.

[16] J. Thorpe, K. Andrews, and S. Dolinar, "Methodologies for designing LDPC codes using protographs and circulants," in Proc. IEEE ISIT, July 2004, pp. 236-236.

[17] D. Divsalar, S. Dolinar, C. R. Jones, and K. Andrews, "Capacity-approaching protograph codes," IEEE J. Select. Areas Commun., vol. 27, no. 6, pp. 876-888, Aug. 2009.

[18] M. El-Khamy, J. Hou, and N. Bhushan, "Design of rate-compatible structured LDPC codes for hybrid ARQ applications," IEEE J. Select. Areas Commun., vol. 27, no. 6, pp. 965-973, Aug. 2009.

[19] T. V. Nguyen, A. Nosratinia, and D. Divsalar, "Bilayer protograph codes for half-duplex relay channels," in Proc. IEEE ISIT, June 2010, pp. 948-952.

[20] G. Liva and M. Chiani, "Protograph LDPC codes design based on EXIT analysis," in Proc. IEEE GLOBECOM, Nov. 2007, pp. 3250-3254.

[21] S. Abu-Surra, D. Divsalar, and W. Ryan, "On the existence of typical minimum distance for protograph-based LDPC codes," in Information Theory and Applications Workshop (ITA), Jan. 2010.

[22] T. Richardson and R. Urbanke, "Multi-Edge Type LDPC Codes,” EPFL, Tech. Rep., 2004, submitted IEEE Trans. Info. Theory.

[23] X.-Y. Hu, E. Eleftheriou, and D.-M. Arnold, "Regular and irregular progressive edge-growth tanner graphs," IEEE Trans. Inform. Theory, vol. 51, pp. 386-398, 2003.

[24] J. Hamkins, "Performance of low-density parity-check coded modulation," IPN Progress Report 42-184, Feb. 2011.

[25] L.-L. Xie and P. Kumar, “An achievable rate for the multiple-level relay channel," IEEE Trans. Inform. Theory, vol. 51, no. 4, pp. 1348 -1358, Apr. 2005.

[26] A. Host-Madsen, "On the capacity of wireless relaying," in Proc. IEEE Vehicular Technology Conference (VTC), vol. 3, 2002, pp. 1333 - 1337.

[27] T. V. Nguyen, A. Nosratinia, and D. Divsalar, "The design of rate-compatible protograph LDPC codes," IEEE Trans. Commun., vol. 60, no. 10, pp. 2841 - 2850, Oct. 2012. 